\title{
Peroral Endoscopic Myotomy, a Well-Established, Efficacious, and Safe Treatment Option for Achalasia: Is the History of Previous Treatment a Hurdle or Not?
}

\author{
Young Hoon Youn \\ Department of Internal Medicine, Gangnam Severance Hospital, Yonsei University College of Medicine, Seoul, Korea
}

See "Experience with Peroral Endoscopic Myotomy for Achalasia and Spastic Esophageal Motility Disorders at a Tertiary U.S. Center" by Maen Masadeh, Peter Nau, Subhash Chandra, et al., on page 321-327.

Recently, peroral endoscopic myotomy (POEM) was introduced as a novel therapeutic modality for the treatment of achalasia and spastic esophageal motility disorders. The first case of achalasia treated with POEM was reported in Japan in 2008. POEM has been widely used by many skillful endoscopists worldwide and is rapidly becoming popular. It involves the application of esophageal myotomy to the concept of natural orifice transluminal surgery by utilizing a submucosal tunneling method and aims at combining the benefits of the endoscopic minimal invasive approach with the good longterm efficacy of Heller myotomy. Clinical outcomes of this new therapeutic option have been reported to be excellent in terms of both efficacy and safety, although some concerns regarding the incidence of postoperative gastroesophageal reflux disease still exist.

In this issue of Clinical Endoscopy, Masadeh et al. reported their experience of 125 cases of achalasia and spastic esophageal motility disorders treated with POEM at a tertiary U.S. center. ${ }^{1}$ This study also showed excellent clinical outcome

Received: April 30, 2020 Revised: May 21, 2020

Accepted: May 21, 2020

Correspondence: Young Hoon Youn

Department of Internal Medicine, Gangnam Severance Hospital, Yonsei University College of Medicine, 211 Eonju-ro, Gangnam-gu, Seoul 06273, Korea Tel: +82-2-2019-3453, Fax: +82-2-3463-3882, E-mail: dryoun@yuhs.ac ORCID: https://orcid.org/0000-0002-0071-229X

(c) This is an Open Access article distributed under the terms of the Creative Commons Attribution Non-Commercial License (http://creativecommons.org/ licenses/by-nc/3.0) which permits unrestricted non-commercial use, distribution, and reproduction in any medium, provided the original work is properly cited.
( $92 \%$ initial clinical success, persisted in $88 \%$ at 12 months), although it included initial cases in which POEM was performed by two endoscopists in their learning curves. Accumulating data, including this report, confirm the excellent clinical efficacy and safety of POEM.

However, this study showed more notable findings than just the excellent clinical outcome, including the significantly higher mucosal barrier failure (MBF) rate in patients with prior laparoscopic Heller myotomy ( $19 \%$ vs. $3 \%, p=0.015) .{ }^{1}$ MBF was defined as a leak of contrast into the submucosal tunnel or mediastinum on postoperative esophagography. The authors discussed that obliteration of the submucosal space by fibrosis and inability to avoid the scarred area may play a role in this finding. Actually, previous endoscopic or surgical treatment may induce submucosal fibrosis at the esophagogastric (EG) junction, hindering safe submucosal tunneling. ${ }^{2}$ Fundoplication accompanied by Heller myotomy may also result in a complex angle of the EG junction. ${ }^{2}$ However, several reports showed similar clinical outcomes and adverse events of POEM for patients with and without prior endoscopic or surgical therapy. ${ }^{3-9}$ These studies argue that previous endoscopic and even surgical treatments are not a contraindication for POEM, ${ }^{2-9}$ and these findings are discordant with those of the report by Masadeh et al. in the current issue. ${ }^{1}$ We need to discuss the reasons for this disagreement.

First, in reality, many POEM endoscopists agree on the higher difficulty of POEM in previously heavily treated achalasia patients. However, accumulated experience and skills enable POEM endoscopists to manage and overcome submu- 
cosal fibrosis. Therefore, there might be a smaller difference in the rate of adverse events on a larger scale in POEM reports. In this study by Masadeh et al., MBF as adverse events mostly occurred early in their clinical experience, so there might be a "learning curve" issue. ${ }^{1}$ Additionally, we must also consider the possibility of "publication bias" because we know that superior and safer clinical outcomes are easier to be accepted for publication.

Second, a recent publication showed findings concordant with those reported by Masadeh et al. in this issue. ${ }^{1}$ Wang et al. reported a large-scale clinical series of 1,912 cases treated with POEM, focusing on mucosal injury (MI) during POEM. ${ }^{10}$ In this study, the authors found that MI was independently associated with previous Heller myotomy (odds ratio [OR], 2.094; $p=0.026)$, previous POEM (OR, $2.441 ; p=0.033)$, submucosal fibrosis (OR, 4.530; $p<0.001$ ), mucosal edema (OR, 1.834; $p=0.001)$, and tunnel length $\geq 13 \mathrm{~cm}(\mathrm{OR}, 2.699 ; p<0.001)$. They also found that POEM experience of $>1$ year was a protective factor for MI (OR, $0.614 ; p=0.042) .{ }^{10}$ This large-scale study showed that previous Heller myotomy and submucosal fibrosis are definite risk factors for MI as an adverse event, and accumulated experience of POEM can play a role as a protective factor.

In case of rescue POEM after previous Heller myotomy, following tips should be noted to overcome the difficulties caused by fibrosis. First, the submucosal tunnel should be made at a different axis from that of the previous myotomy, to avoid the submucosal fibrosis and scar regions of the previous myotomy. Therefore, a meticulous review of previous surgical notes is mandatory. Because in most cases, Heller myotomy is performed in the anterior aspect, rescue POEM is generally recommended for approach in the posterior aspect of the esophagus. Second, if submucosal fibrosis is too broad to be avoided, submucosal tunneling should be performed as deep as possible. While damage to the muscularis propria during tunneling does not significantly affect the outcome, mucosal damage from electrocoagulation or a mucosal tear can often cause adverse events. Moreover, the use of cutting current rather than the spray coagulation mode of an electrosurgical unit may help reduce electrocoagulation damage to the mucosal layer in highly fibrotic areas. Third, rescue POEM should be performed by fully experienced POEM endoscopists because it is often technically demanding compared to standard POEM. Onimaru et al. recommended an experience of at least 30 cases of standard POEM before attempting rescue POEM based on their study. ${ }^{2}$

To conclude this commentary, POEM has been rapidly becoming popular worldwide owing to its excellent clinical outcomes in a short period of time, and indications for POEM are expanding to include long-standing sigmoid-shaped achalasia, other spastic esophageal motility disorders, and even previously failed endoscopic treatment or surgical myotomy cases. However, rescue POEM after previous Heller myotomy is often highly technical, so it may be better performed by a fully experienced POEM endoscopist. A study from the U.S. by Masadeh et al. also showed excellent clinical outcomes of POEM and gives us a chance to learn how to reduce the adverse events of rescue POEM. ${ }^{1}$

\section{Conflicts of Interest}

The author has no financial conflicts of interest.

\section{REFERENCES}

1. Masadeh M, Nau P, Chandra S, et al. Experience with peroral endoscopic myotomy for achalasia and spastic esophageal motility disorders at a tertiary U.S. center. Clin Endosc 2020;53:321-327.

2. Onimaru $\mathrm{M}$, Inoue $\mathrm{H}$, Ikeda $\mathrm{H}$, et al. Peroral endoscopic myotomy is a viable option for failed surgical esophagocardiomyotomy instead of redo surgical Heller myotomy: a single center prospective study. J Am Coll Surg 2013;217:598-605.

3. Ling T, Guo H, Zou X. Effect of peroral endoscopic myotomy in achalasia patients with failure of prior pneumatic dilation: a prospective case-control study. J Gastroenterol Hepatol 2014;29:1609-1613.

4. Tang X, Gong W, Deng Z, et al. Feasibility and safety of peroral endoscopic myotomy for achalasia after failed endoscopic interventions. Dis Esophagus 2017;30:1-6.

5. Orenstein SB, Raigani S, Wu YV, et al. Peroral endoscopic myotomy (POEM) leads to similar results in patients with and without prior endoscopic or surgical therapy. Surg Endosc 2015;29:1064-1070.

6. Nabi Z, Ramchandani M, Chavan R, et al. Peroral endoscopic myotomy in treatment-naïve achalasia patients versus prior treatment failure cases. Endoscopy 2018;50:358-370.

7. Jones EL, Meara MP, Pittman MR, Hazey JW, Perry KA. Prior treatment does not influence the performance or early outcome of per-oral endoscopic myotomy for achalasia. Surg Endosc 2016;30:1282-1286.

8. Sharata A, Kurian AA, Dunst CM, Bhayani NH, Reavis KM, Swanström LL. Peroral endoscopic myotomy (POEM) is safe and effective in the setting of prior endoscopic intervention. J Gastrointest Surg 2013;17:11881192.

9. Liu ZQ, Li QL, Chen WF, et al. The effect of prior treatment on clinical outcomes in patients with achalasia undergoing peroral endoscopic myotomy. Endoscopy 2019;51:307-316.

10. Wang Y, Liu ZQ, Xu MD, et al. Clinical and endoscopic predictors for intraprocedural mucosal injury during per-oral endoscopic myotomy. Gastrointest Endosc 2019;89:769-778. 